\title{
Linfangioleiomiomatosis pulmonar: Caso clínico
}

\author{
Rafael Silva 0 1,2,7, Felipe Puelma $C^{3,6}$, Víctor Retamal P5, \\ Pedro Rojas SM ${ }^{4,6}$, Claudio Cruzat $C^{6}$, Cecilia Reyes $\mathbf{G}^{1 \mathrm{a}}$. \\ Pulmonary lymphangioleiomyomatosis: \\ Report of one case
}

Lymphangioleiomyomatosis (LAM) is a rare interstitial lung disease, of unknown etiology, affecting almost exclusively women. Microscopically, LAM consists of a diffuse proliferation of smooth muscle cells. LAM can occur without evidence of other diseases (sporadic LAM) or in conjunction with tuberous sclerosis complex (TSC). It presents with progressive breathlessness or with recurrent pneumothorax or chylothorax. We report a 33 year-old woman with a history of recurrent pneumothorax. Computed tomography (CT) scans showed numerous thin-walled cysts throughout the lungs, a characteristic finding in LAM. A pulmonary biopsy was compatible with the diagnosis and HMB- 45 monoclonal antibodies were positive. Treatment with Sirolimus was started (Rev Méd Chile 2009; 137: 1474-7).

(Key words: Lymphangioleiomyomatosis; Lung diseases; Pneumothorax)

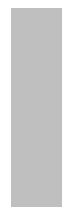

\begin{abstract}
Recibido el 30 de diciembre, 2008. Aceptado el 7 de septiembre, 2009.
${ }^{1}$ Unidad de Enfermedades Respiratorias, ${ }^{2}$ Servicio de Medicina, ${ }^{3}$ Servicio de Cinugía y ${ }^{4}$ Servicio de Ginecología, Hospital Regional de Talca. ${ }^{5}$ Servicio de Radiología, Hospital Base de Linares. 6Unidad de Patología, Escuela de Medicina, Universidad de Talca. Unidad de Anatomía, Escuela de Medicina, Universidad Católica del Maule. ${ }^{7}$ Escuela de Medicina, Universidad de Talca. aEnfermera Universitaria
\end{abstract}

L infangioleiomiomatosis pulmonar (LAM) es una enfermedad intersticial idiopática de muy baja ocurrencia que afecta predominantemente a mujeres en edad fértil que fue descrita por primera vez en $1937^{1}$. Se caracteriza por una proliferación anormal de músculo liso en pulmón, de aspecto benigno y de predominio peribroncovascular, que provoca obstrucción de linfáticos, vasos sanguíneos y bronquiolos ${ }^{2}$. Ello contribuye a la formación de quistes por obstrucción de la pequeña vía aérea, cuya rotura provoca neumotórax recidivante, aun cuando algunos estudios han señalado que la degradación de fibras elásticas podría estar involucrada en la formación de estos

Correspondencia a: Dr. Rafael Silva O. Dos Norte 360 Talca. Fono/Fax: (71) 231616. E mail: rafaelsilvao@gmail.com quistes $^{3}$. Esta enfermedad afecta preferentemente a mujeres, fundamentalmente en edad fértil, con una media de edad en el momento de la presentación de 30 a 35 años, estimándose una incidencia entre 2 a 5 casos por millón de mujeres, asociándose en algunos casos al complejo esclerosis tuberosa en $40 \%$ y a angiomiolipomas renales.

\section{CASO CLÍNICO}

Mujer de 33 años, profesora de Educación Física, sin antecedentes de patologías respiratorias, neurológicas ni dermatológicas. Sin antecedentes tabáquicos y sin limitaciones al ejercicio. En abril del año 2003 presentó aborto espontáneo de 12 semanas. En julio de 2003 consultó por dolor torácico y apremio respiratorio. Se constató neumotórax dere- 
cho, con tomografía computada de tórax (Figuras 1a-1b) que mostró imágenes cistoideas escasas bilaterales. Se selló la pleura derecha con talco y fue dada de alta en buenas condiciones.

El año 2004 cursó embarazo, con parto vaginal en diciembre de ese año, sin incidentes. Se mantuvo asintomática hasta marzo de 2007, en que presentó un neumotórax izquierdo, tratado con sellamiento pleural izquierdo y fue dada de alta en buenas condiciones. No se realizó tomografía computada de tórax en esa oportunidad.

El 3 de mayo de 2008 presentó nuevamente un neumotórax derecho. La tomografía computada pulmonar mostró múltiples imágenes cistoideas, de pared fina, difusas, francamente progresivas respecto a tomografía del año 2003 (Figuras 1c-1d).

Se realizó drenaje pleural derecho y videotoracoscopia, detectándose un pulmón difusamente eritematoso con múltiples lesiones quísticas. Se efectuó sellamiento con talco y se practicó biopsia pulmonar que describió parénquima pulmonar con arquitectura en parte conservada y áreas focales caracterizadas por espacios alveolares dilatados y confluentes, con rotura parcial de tabiques y proliferación fasciculada y pseudonodular de células fusadas mesenquimáticas de tipo músculo liso, inmaduras, sin atipias, dispuestas en paredes bronquiolo-alveolares y en parte perivasculares. Sin lesiones granulomatosas ni signos de malignidad, altamente sospechosa de linfangioleiomiomatosis pulmonar (Figuras 2a-2b).

Se realizó estudio inmunohistoquímico que mostró:

a) HMB-45 (+) tinción citoplasmática en células fusadas (Figuras 2c-2d). b) Actina músculo liso (+) tinción citoplasmática en células fusadas. c) Receptor estrógeno (+) tinción nuclear en 60\%-70\% de células fusadas. d) Receptor de progesterona (+) tinción nuclear en 30\%-40\% de células fusadas. e) Tinción histoquímica de orceína: disnupción parcial de elastina en paredes vasculares; concordantes con linfangioleiomiomatosis pulmonar con receptores hormonales (+). Además se comprueba Alfa-1-antitripsina normal.

En agosto de 2008 se realizó espirometría y pletismografía, ambas normales y prueba de caminata de $6 \mathrm{~min}$, la distancia caminada estuvo dentro del valor predictivo, sin caída de la saturación de oxígeno.

Fue evaluada por ginecólogo quien indica bloqueo hormonal con triptorelina 3,75 mg cada mes (Decapeptil ${ }^{\circledR}$ ), análogo de $\mathrm{Gn}$ - $\mathrm{RH}$, para provocar hipoestrogenismo hipogonadotrófico reversible y prevención de osteoporosis con ibandronato 150 mg oral mensual (Idena ${ }^{\circledR}$ ) más aporte de calcio oral. El neumólogo tratante recomendó iniciar tratamiento empírico, con Sirolimus (Rapamune ${ }^{\circledR}$ ) $2 \mathrm{mg}$ por día, entregando la información científica disponible, lo que fue aceptado por la paciente y su esposo. Estos tratamientos comenzaron en agosto de 2008 con control seriado de niveles plasmáticos de Sirolimus, hemograma y perfil lipídico.

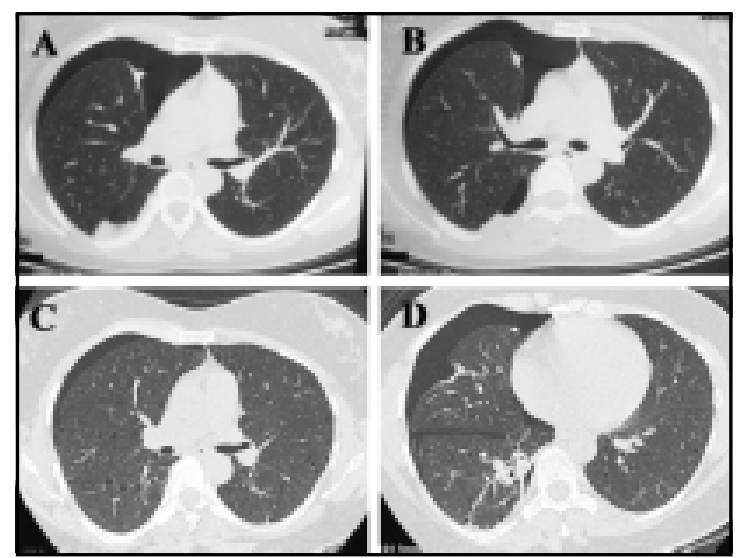

Figura 1. Tomografía computada tórax, julio 2003. A) y B) Neumotórax derecho y escasas lesiones quísticas difusas. Tomografía computada tórax, mayo 2008: C) y D) Aumento progresivo de lesiones quísticas difusas y nuevo neumotórax derecho.

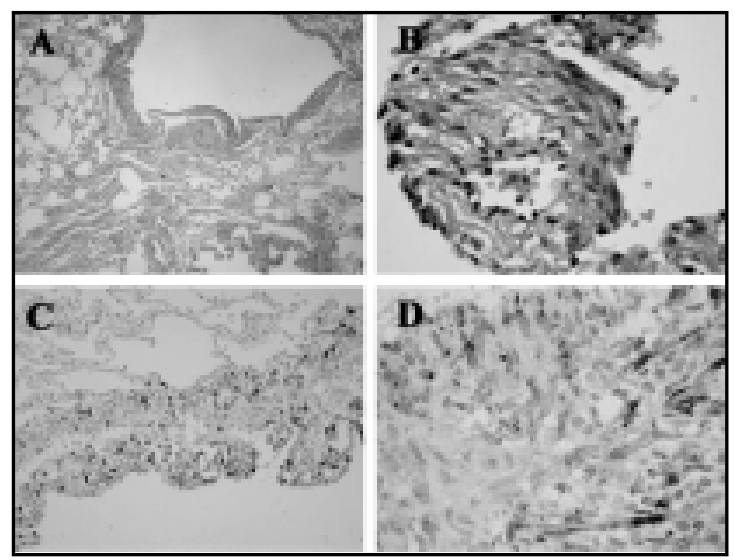

Figura 2. Biopsia pulmonar: A) y B) Proliferación fasciculada y pseudonodular de células fusadas de tipo músculo liso (células LAM) dispuestas en paredes bronquiolo-alveolares y perivasculares. Hematoxilinaeosina. C) y D) Estudio inmunohistoquímico con anticuerpos monoclonales, tinción HMB-45 positiva en células fusadas de tipo músculo liso (células LAM). 


\section{DisCUSIÓN}

LAM se presenta como una enfermedad pulmonar intersticial, quística, difusa, progresiva, con neumotórax recurrentes, quilotórax y finalmente, falla respiratoria progresiva.

Desde el punto de vista patológico las células mesenquimáticas fusadas atípicas de músculo liso, llamadas "células LAM", se acumulan progresivamente en los pulmones y en los linfáticos con características no típicas de una célula de músculo liso normal. Estas contienen proteínas relacionadas al melanoma, incluyendo glicoproteína 100, el gatillo de HMB-45 (Antibody Human Melanoma Black) 4,5 y receptores para estrógenos y progesterona ${ }^{6}$. Estas células tienen una alta capacidad proliferativa, dañando la vía aérea a través de la producción de metaloproteinasas, junto con, como ya se mencionó, rodear y obstruir los capilares, los linfáticos y la vía aérea7. La obstrucción linfática suele provocar quilotórax y la obstrucción de la vía aérea condiciona un patrón funcional obstructivo. El 30\%-40\% de los casos presenta neumotórax, que puede ser bilateral y recurrente ${ }^{10}$, como el caso en presentación.

Durante largos periodos los pacientes están asintomáticos y cuando aparecen síntomas, como la disnea, la enfermedad está en etapa avanzada. Se describen angiomiolipomas renales hasta en $40 \%$ de los casos 8 .

El hallazgo característico en la tomografía computada de tórax es la presencia de innumerables quistes de paredes finas rodeados por parénquima pulmonar normal, que presentan distribución uniforme, sin predilección por alguna zona. Generalmente miden menos de $2 \mathrm{~cm}$ de diámetro y el grosor de su pared varía desde prácticamente imperceptible hasta $4 \mathrm{~mm}$. Generalmente no existen líneas septales interlobulillares engrosadas ni destrucción vascular?

La distribución uniforme de los quistes en ambos pulmones apoya significativamente el diagnóstico de la enfermedad y ayuda a distinguir la LAM de otras enfermedades pulmonares quísticas difusas, particularmente de la enfermedad de Langerhans, que afecta a los dos tercios superiores respetando relativamente las bases. La histiocitosis $\mathrm{X}$ se asocia, además, a nódulos pulmonares, hallazgo raramente asociado a la LAM ${ }^{11}$.

Existe asociación entre LAM y el complejo esclerosis tuberosa (TSC), el cual es una enfermedad neurocutánea autosómica dominante, ligada a dos genes supresores de tumores: TSC1 y TSC2. Estos genes codifican dos proteínas: hamartina (TCS1) y tuberina (TSC2), que son relevantes en regular el tamaño y el crecimiento celular. Las proteínas TSC1 y TSC2 regulan su señal a través de mTOR (mammalian target of rapamycin), controlando el crecimiento celular, el ciclo celular, la apoptosis y la autofagia. La activación de mTOR provoca las lesiones que se observan en la esclerosis tuberosa y en la LAM $^{12,13}$.

Se observan tres situaciones clínicas: a) LAM aislado (S-LAM) que no tiene características clínicas de TSC, b) S-LAM con angiomiolipomas renales y c) pacientes con complejo esclerosis tuberosa en los cuales se asocia una LAM (TSC-LAM). Nuestra paciente presenta la primera situación. El diagnóstico definitivo en LAM se realiza generalmente por confirmación histológica e inmunohistoquímica, con tinción positiva del anticuerpo monoclonal HMB-45 en el tejido obtenido por punción-aspiración, biopsia transbronquial o por biopsia pulmonar a cielo abierto, como el caso que se comenta. El hallazgo de HMB-45 es actualmente considerado el patrón de oro para el diagnóstico de LAM, por su alta sensibilidad y especificidad ${ }^{14}$.

Dado que, tanto por técnicas inmunohistoquímicas como bioquímicas, se han identificado receptores para estrógenos y progesterona en el tejido pulmonar de las pacientes con LAM, se preconizó el tratamiento con ooforectomía y actualmente con bloqueo estrogénico, aun cuando la presencia de estos receptores no esté claramente asociada a una mejor respuesta al tratamiento hormonal.

Pese a lo anterior, en mujeres premenopáusicas, el manejo actual recomienda terapia antiestrogénica, basado en algunos reportes de casos y pequeñas series clínicas ${ }^{15}$.

En ausencia de buena evidencia no se recomienda el uso rutinario de progesterona o agonistas de las gonadotropinas. También se ha empleado tamoxifeno como tratamiento cuyos resultados no son claros respecto algún beneficio. Asimismo se ha comunicado el uso de agonistas de gonadotrofina (Gn-RH) como la triptorelina, con resultados contradictorios, aun cuando está demostrado que su uso no revierte la declinación del $\mathrm{VEF} 1^{16}$. El tratamiento con ooforectomía no se recomienda actualmente.

Se ha observado que la LAM es exacerbada por embarazo y por uso de terapia estrogénica exógena ${ }^{17}$ por lo cual, obviamente, se debe 
desaconsejar el embarazo y el uso de estrógenos. En los casos avanzados y con daño funcional grave, se recomienda el trasplante pulmonar ${ }^{18}$.

Está en curso un protocolo placebo-controlado con Sirolimus (Rapamicina), que, como se explicó previamente, corrige las anormalidades celulares producidas por la pérdida de función de TSC1/TSC2, al ser Sirolimus un inhibidor de mTOR. Se espera demostrar que este medicamento disminuye la progresión del daño funcional pulmonar. Los resultados de este protocolo denominado MLES, estarán disponibles el año 2011. Se trata de un estudio clínico fase II, cuyo objetivo principal es prevenir la caída de la función pulmonar en pacientes con LAM ${ }^{19}$.

El pronóstico de esta enfermedad históricamente ha sido malo, pero ha mejorado en los

\section{REFERENCIAS}

1. Von Stossel E. Uber Musculature Cirrhose der lunge (Muscular Cirrhosis of de lungs). Beitr Klin Tuberk 1937; 90: 432-42.

2. Jonson SR. Lymphangioleiomyomatosis. Eur Respir J 2006; 27: 1056-65.

3. Matsui $K$, Takeda K, Yu ZX, Zu-XI Y, Travis WD, Moss J, FeRRANS VJ. Role for activation of matrix metalloproteinases in the pathogenesis of pulmonary lymphangioleiomyomatosis. Arch Pathol Lab Med 2000; 124: 267-75.

4. TANAKA H. Diagnosis of pulmonary lymphangioleiomyomatosis by HMB45 in surgically treated spontaneous pneumothorax. Eur Respir J 1995; 8: 1879-82.

5. Matsumoto Y, Horiba K, Usuki J, Chu SC, Ferrans VJ, Moss J. Markers of cell proliferation and expression of melanosomal antigen in lymphangioleiomyomatosis. Am J Respir Cell Mol Biol 1999; 21: 327-36.

6. BANNER AS. Hormone receptors in lymphangioleiomyomatosis. Chest 1984; 85: 3-4.

7. Zhe $\mathrm{X}, \mathrm{Y}_{\text {ang }} \mathrm{Y}$, Jakkaraju S, Schuger L Tissue inhibitor of metalloproteinase-3 downregulation in lymphangioleiomyomatosis: potential consequence of abnormal serum response factor expression. Am J Respir Cell Mol Biol 2003; 28: 504-11.

8. JohNSON S. Lymphangioleiomyomatosis: clinical features, management and basic mechanisms. Thorax 1999; 54: 254-64.

9. Cosgrove G, Frankel S, Brown K. Challenges in Pulmonary Fibrosis. 3: Cystic lung disease. Thorax 2007; 62: 819-28.

10. Steagall W, Giasgow C, Hathaway O, Avita N, Taverra-Da Silva A, RABEL A ET AL. Genetic and mophologic determinants of pneumothorax in lymphangioleiomyomatosis. Am J Physiol Lung Cell Mol Physiol 2007; 293: 800-8. últimos años, aparentemente con los tratamientos hormonales usados, pero existen sólo datos anecdóticos respecto a que estos tratamientos puedan mejorar el curso natural de la enfermedad y el pronóstico de estas pacientes ${ }^{20}$.

En resumen, la presencia de neumotórax recurrente, en una mujer en edad reproductiva debe hacernos sospechar la posibilidad de LAM particularmente si la tomografía computada de tórax muestra lesiones quísticas difusas, y su estudio funcional muestra patrón obstructivo. El diagnóstico debe confirmarse con biopsia pulmonar que incluya técnicas de inmunohistoquímica por cuanto la tinción positiva del anticuerpo monoclonal HMB-45 en el tejido, tiene alta sensibilidad y especificidad para el diagnóstico de $\mathrm{LAM}^{14}$.

11. Attili A, Kazerooni E. Case 116: Lymphangioleiomyomatosis. Radiology 2007; 244: 303-8.

12. Schwartz R, Fernández $G$, Kotulska $K$, Jozwiak $S$. Tuberous sclerosis complex: Advances in diagnosis, genetics, and management. J Am Acad Dermatol 2007; 57: 189-202.

13. Crino P, Nathanson K, Henske E. The Tuberous Sclerosis Complex. N Engl J Med 2006; 355: 1345-56.

14. Tanaka H, Imada A, Morikana T, Shibusa T, Satoh M, SEKINE K ET AL. Diagnosis of pulmonary lymphangioleiomyomatosis by HMB45 in surgically treated spontaneous pneumothorax. Eur Respir J 1995; 8: 1879-82.

15. Taveira-Da Silva A, Stylianou MP, Hedin CJ, Hathaway $\mathrm{O}$, Moss J. Decline in lung function in patients with lymphangioleiomyomatosis treated with or without progesterone. Chest 2004; 126: 1867-74.

16. Harari S, Cassandro R, Chiodini J, Taveira-Da Silva A, Moss J. Effect of a Gonadotrophin-Releasing Hormone Analogue on Lung Function in Lymphangioleiomyomatosis. Chest 2008; 133: 448-54.

17. Yamashita C, Lynch D, Downey G. Lymphangioleiomyomatosis. Clin Pulm Med 2008; 15: 325-31.

18. Maurer JR, Ryu J, Beck G, Moss J, Lee J, Finlay G et al. Lung transplantation in the management of patients with lymphangioleiomyomatosis: baseline data from the NHLBI LAM Registry. J Heart Lung Transplant 2007; 26: 1293-9.

19. McCORMACK F. Multicenter International Lymphangioleiomyomatosis Efficacy of Sirolimus Trial, Disponible en: http://clinicaltrials.gov/ct2/show/NCToo720746?cond =\%22Lymphangioleiomyomato-sis\%22\&rank=2 [Consultado el 8 de diciembre, 2008].

20. Kтtachi M, Nishimura K, Itoh H, Izumi T. Pulmonary lymphangioleiomyomatosis: a report of 46 patients including a clinicopathologic study of prognostic factors. Am J Respir Crit Care Med 1995; 151 (2 pt 1): 527-33. 